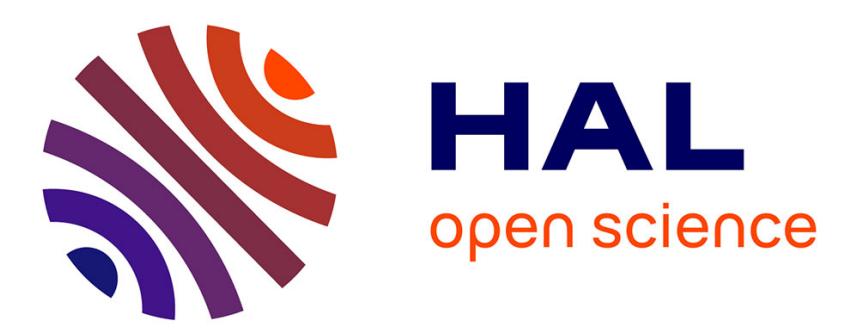

\title{
Représentation mentale et processus moteur: le cas de la rotation mentale
}

\author{
Nady Hoyek, Christian Collet, Aymeric Guillot
}

\section{To cite this version:}

Nady Hoyek, Christian Collet, Aymeric Guillot. Représentation mentale et processus moteur: le cas de la rotation mentale. Science \& motricité: Revue scientifique de l'Association des Chercheurs en Activités Physiques et Sportives, 2010, 71 (71), pp.29-39. 10.1051/sm/2009013 . hal-01743960

\section{HAL Id: hal-01743960 \\ https://univ-lyon1.hal.science/hal-01743960}

Submitted on 26 Mar 2018

HAL is a multi-disciplinary open access archive for the deposit and dissemination of scientific research documents, whether they are published or not. The documents may come from teaching and research institutions in France or abroad, or from public or private research centers.
L'archive ouverte pluridisciplinaire HAL, est destinée au dépôt et à la diffusion de documents scientifiques de niveau recherche, publiés ou non, émanant des établissements d'enseignement et de recherche français ou étrangers, des laboratoires publics ou privés. 


\title{
Représentation mentale et processus moteur : le cas de la rotation mentale
}

\author{
N. Hoyek, C. Collet et A. Guillot \\ Université Claude Bernard Lyon 1, Université de Lyon, EA 647, Centre de Recherche et d'Innovation sur le Sport, \\ Laboratoire de la Performance Motrice, Mentale et du Matériel, 27-29 boulevard du 11 novembre 1918, \\ 69622 Villeurbanne Cedex, France
}

Reçu le 7 novembre 2008 - Accepté le 2 février 2009

\begin{abstract}
Résumé. La Rotation mentale $(\mathrm{RM})$ est une capacité qui consiste à faire tourner mentalement une image en 2 ou en 3 dimensions. Ses relations avec les processus moteurs restent controversées. Entre stratégie interne ou égocentrique et stratégie externe ou visuelle, entre activation des régions motrices et des régions visuelles, les résultats restent contradictoires. La question de l'activation des régions motrices attestant des processus moteurs nécessaires à la résolution des problèmes de RM, ou simple reflet de la planification et l'exécution d'une réponse motrice n'a pas encore reçu de réponse définitive. On ne sait pas non plus précisément si la capacité de RM est liée à l'expérience professionnelle, motrice, ou sportive. L'objectif de ce travail est de faire la synthèse des publications présentant des données obtenues par imagerie cérébrale ou des données comportementales (analyse des temps de réponse, par exemple) ou alors impliquant différents stimuli (cubes de Shepard et Metzler, chiffres, lettres, segments corporels, formes géométriques), ou encore différentes stratégies mentales (interne ou externe).
\end{abstract}

Mots clés : Rotation mentale, processus moteurs, imagerie cérébrale, stratégie interne/externe

\begin{abstract}
Mental representation and motor process: the mental rotation case.
Mental Rotation (MR) is the ability to form a mental image of a $2 \mathrm{D}$ or 3D object turning in space. The relationships with motor process still remain unclear. Inconsistent results were probably due to the use of at least, two different strategies by the participants, i.e. internal or egocentric strategy versus external or visual strategy which resulted in different patterns of cerebral activations (motor region and visual regions, respectively). Does the motor regions activation reflect a motor process required for the resolution of $\mathrm{MR}$ problems? Or is it due to the simple planning and execution of a motor response? Is MR ability linked to professional, motor or self-experience in sport? The aim of this article is to review and analyze the main studies involving different methodological paradigms (cerebral imagery, behavioral studies), and using different stimuli (Shepard and Metzler cubes, alphanumeric characters, body parts, geometric drawings) as well as studies comparing internal and external strategies.
\end{abstract}

Key words: Mental rotation, motor process, cerebral imagery, internal/external strategy

\section{Introduction}

La rotation mentale $(\mathrm{RM})$ est la capacité à faire tourner mentalement l'image d'un objet en 2 ou en 3 dimensions. Il s'agit d'une forme particulière d'imagerie mentale ou motrice, qui nécessite une structuration de l'espace et implique parfois les processus moteurs. Typiquement, ces représentations mentales nécessitent de former une image visuelle, même si certains types d'imagerie peuvent épisodiquement ne pas inclure la vision, comme en témoignent des réseaux d'activations cérébrales spécifiques (voir Guillot et al., 2009). Toutefois, la qua- lité de la représentation renvoie à la vivacité (netteté) de l'image formée, à son exactitude (fidélité par rapport à la réalité) et à son contrôle (capacité à la manipuler et la transformer). Cette dernière dimension nécessite alors de maîtriser la RM, pour visualiser l'image, l'objet ou le mouvement selon différents angles, ce qui peut nécessiter l'implication des processus moteurs. Une bonne représentation mentale requiert donc la capacité à travailler l'image mentale visuelle, à la faire tourner mentalement.

La tâche de RM la plus connue et la plus fréquemment utilisée consiste à indiquer le plus rapidement possible, si 
deux images en 2 ou 3 dimensions (2D ou 3D), présentées sous différents angles, sont identiques ou différentes. Ce problème nécessite d'effectuer une RM d'au moins une des figures dans un des plans de l'espace, afin de la superposer avec l'autre pour juger de leur disparité ou similitude. Une autre stratégie, sans doute plus difficile à utiliser pour comprendre les mécanismes impliqués dans la RM, consisterait à s'imaginer se déplacer soi-même en tournant autour de l'objet afin de le visualiser sous un angle différent. Les travaux de Shepard, dès 1970 (Shepard \& Metzler, 1971; Cooper \& Shepard, 1973; Metzler \& Shepard, 1974) constituent les études pionnières dans ce domaine. Shepard \& Metzler (1971) ont ainsi constaté que, plus la disparité angulaire entre les figures était grande, plus le temps de réponse pour comparer les structures augmentait.

S'inspirant de Shepard \& Metzler (1971), Vandenberg \& Kuse (1978) ont validé un test papier-crayon basé sur le même principe (cubes Shepard et Metzler ou S-M cubes). À ce jour, l'un des tests les plus utilisés pour estimer les capacités individuelles de RM est le VMRT (Vandenberg and Kuse Mental Rotation test). Il comporte 24 items de 5 figures : la première est le modèle placé à gauche de la ligne, avec 4 figures placées à sa droite, mais sous des angles différents et dont 2 seulement sont identiques à la référence. Les sujets doivent indiquer lesquelles dans un temps imparti de 6 minutes. Vandenberg \& Kuse (1978) ont proposé d'attribuer 1 point seulement quand les deux bonnes figures avaient été repérées et 0 point dans tous les autres cas possibles.

La capacité de RM apparait très tôt durant l'enfance. Selon Marmor (1975, 1977), elle serait effective dès l'âge de 4 ans, mais s'affaiblirait par la suite, chez les sujets vieillissants. En effet, ces derniers obtiennent généralement des scores plus faibles que les jeunes adultes aux tests de RM (Hertzog et al., 1993; Dror \& Kosslyn, 1994; Band \& Kok, 2000). Comme nous le verrons par la suite, plusieurs publications ont également fait état d'un effet du genre, les hommes obtenant de meilleurs scores que les femmes (Linn \& Petersen, 1985; Peters et al., 1995; Peters, 2005; Vandenberg \& Kuse, 1978; Voyer et al., 1995; Guillot et al., 2007a).

Plusieurs expériences ont ensuite cherché à comprendre les mécanismes sous-jacents de la RM, ses liens avec la performance et l'expérience motrice (Naito, 1994; Ozel et al., 2004; Guillot et al., 2007b). On s'est également interrogé sur l'évolution des capacités individuelles de RM à la suite d'un entraînement (Alington et al., 1992; Kass et al., 1998; Roberts \& Bell, 2000; Hoyek et al., 2009) et son transfert vers d'autres domaines d'expertise (Rochford, 1985; Garg et al., 2001; Hegarty et al., 2008; Wanzel et al., 2007; Guillot et al., 2007a; Hoyek et al., 2009). Pour cela, d'autres formes de tests de RM ont été utilisées, impliquant des lettres et des chiffres (Vingerhoets et al., 2001) et même des segments corporels (Parsons, 1994).
Une partie importante de la littérature a ainsi été consacrée à l'étude des bases neurofonctionnelles de la RM à l'aide de l'imagerie cérébrale, par résonance magnétique (IRMf), tomographie par émission de positrons (TEP), magnétoencéphalographie (MEG) ou électroencéphalographie (EEG).

Cette revue de question a donc pour objectif de synthétiser les connaissances liées à la RM et à établir ses rapports avec la motricité, sous l'angle des différentes théories qui ont été proposées dans la littérature.

\section{Rotation mentale et processus moteurs}

La RM nécessite la construction, la transformation et la manipulation d'une image visuelle. En ce sens, elle se construit sur l'image mentale. Elle implique également des processus moteurs pour la mettre en mouvement et la faire tourner. Selon le protocole expérimental, la tâche et le stimulus, un sujet pourrait s'imaginer en être l'acteur. Dans cette partie, nous décrirons les bases neurofonctionnelles de la RM et les principaux résultats obtenus par imagerie cérébrale. La RM de segments corporels ou d'outils manipulables sollicite particulièrement la motricité. Une troisième sous-partie sera consacrée aux théories expliquant la RM : la stratégie interne est égocentrique et implique la motricité; la seconde, externe, est utilisée pour exécuter la rotation mentale et est plutôt visuelle. Une quatrième et dernière partie exposera une série d'études comportementales sur l'interférence entre $\mathrm{RM}$ et rotation manuelle simultanées. Ces études-ci viendront appuyer la théorie selon laquelle la RM est une forme de simulation motrice.

\subsection{Bases neurofonctionnelles de la RM}

D'une manière générale, la neuroimagerie fonctionnelle a montré que la RM activait principalement le cortex pariétal supérieur, les secteurs visuels extrastriés et les régions motrices et/ou pré-motrices. Dans une récente revue de questions, Zacks (2008) le confirme en précisant que l'activation est bilatérale bien que plus marquée dans le cortex pariétal droit et frontal gauche. Le cortex pariétal inferieur gauche serait aussi activé durant la RM de figures abstraites (Alivisatos \& Petrides, 1996). Cohen et al. (1996) n'ont néanmoins pas identifié de dominance hémisphérique et les stratégies cognitives adoptées sont variables d'une personne à l'autre. C'est sans doute pour cette raison qu'ils ont observé que les régions motrices n'étaient pas activées chez tous les sujets, l'aire 6 l'étant chez la moitié seulement. Les aires oculomotrices et les régions pariétales supérieures (notamment le pré-cuneus) seraient activées lorsqu'on doit faire l'analyse descriptive des figures imaginées mentalement, sans doute en raison des exigences visuospatiales de la tâche. Enfin, l'activation des aires occipitales associatives et plus particulièrement de V5/MT, attesterait la visualisation d'un objet en mouvement. 
Les corrélats neuroanatomiques de la RM chez les enfants ont fait l'objet de multiples recherches expérimentales. En particulier, les chercheurs ont étudié les configurations d'activations cérébrales chez les enfants et les adultes. Kucian et al. (2007) ont, par exemple, observé une activation de l'aire pariétale droite chez les enfants, plus particulièrement dans le sillon interpariétal alors qu'elle serait bilatérale chez les adultes. Ils ont conclu que cette évolution était due à l'effet de la maturation et de l'expertise en précisant que l'hémisphère droit interviendrait lors de l'acquisition de nouvelles capacités alors que l'hémisphère gauche serait plutôt activé pour des tâches cognitives déjà acquises. Au contraire, Jansen-Osmann \& Heil (2007), ainsi que Heil \& JansenOsmann (2007), ont rapporté une activation de l'aire pariétale gauche chez les enfants et une activation bilatérale chez les adultes. Selon eux, cette différence serait le reflet de l'évolution d'un raisonnement « analytique » (rotation d'une partie du stimulus) chez l'enfant, à un raisonnement « holistique $»$ (rotation du stimulus dans son intégralité) chez l'adulte. Ces différences dans les résultats expérimentaux peuvent être dues, d'après Kucian et al. (2007), à l'utilisation de différents types de RM et de stimuli d'une étude à l'autre.

Cependant, il n'y a pas consensus sur l'implication des régions motrices. Plusieurs auteurs (Harris et al., 2000; Iwaki et al., 1999; Jordan et al., 2001; Richter et al., 1997) font état d'une activation du lobule pariétal supérieur et du sillon intra pariétal, parfois associée à celle des régions visuelles (Barnes et al., 2000; Harris et al., 2000 ; Jordan et al., 2001). En revanche, ils n'ont pas observé d'activation des régions motrices. Au contraire, Richter et al. (2000) ont observé une activation bilatérale des aires pré-motrice et motrice supplémentaire durant la RM de S-M cubes, de même que Vingerhoets et al. (2001) ont identifié celle du cortex moteur primaire lors de la RM de lettres et de chiffres, sans que ces derniers n'avancent de justification à cela. Ils ont cependant confirmé l'activation des régions pariétales supérieures lors de la RM de figures abstraites. La RM impliquerait donc un ensemble de processus cognitifs et le simple changement du stimulus peut amener à des configurations d'activation cérébrale différentes. Windischberger et al. (2003) ont aussi montré que les aires pariétales et pré-motrices sont les principales activées. Plus récemment Lamm et al. (2007) ont décrit l'activité cérébrale au cours des différentes phases de la RM : la phase durant laquelle le sujet voit apparaître le stimulus sur l'écran et reconnaît ses contours et orientation est l'encodage. La RM suit et transforme le stimulus spatialement grâce à sa représentation mentale. Enfin, le sujet compare le stimulus tourné mentalement à la référence pour juger de leur éventuelle disparité et appuie sur un bouton pour répondre. Lamm et al. (2007) ont rapporté une activation du cortex prémoteur dorsolatéral qui ne serait pas en relation avec le contrôle du mouvement oculaire et serait indépendante de la quantité d'informations spatiales à analyser. Elle correspondrait à des processus plus généraux comme l'attention visuo-spatiale et l'anticipation du mouvement. Son activation suggère que la RM dépend d'un processus visuo-moteur considéré comme une simulation d'une rotation réelle d'un objet. Lamm et al. (2007) ont précisé que c'est la région rostrale (PMdr) qui était la plus activée. Selon eux, la partie caudale (PMdc) serait davantage impliquée dans l'imagerie motrice de l'objet ne nécessitant pas d'être manipulé ou transformé. En revanche le PMdr n'est pas connecté aux régions impliquées dans l'exécution motrice mais avec celles du cortex préfrontal, ce qui supposerait que le PMdr est plus impliqué dans un processus cognitif que moteur. On peut alors conclure que RM et imagerie motrice activent des circuits nerveux spécifiques, et sont deux processus complémentaires, la RM restant une forme particulière d'imagerie motrice.

D'après Zacks (2008), le cortex pariétal supérieur contrôle les transformations visuo-spatiales des images car la RM est une représentation spatiale durant laquelle les sujets visualisent une rotation. Cependant l'activation des régions motrices varie d'une étude à l'autre. La majorité des résultats mentionne le gyrus précentral, impliqué dans la planification et l'exécution motrice. Or Zacks (2008) s'est demandé si cette activation motrice atteste la simulation motrice nécessaire à l'exécution de la RM ou simplement la planification et l'exécution de la réponse (en général, il faut appuyer sur un bouton en fin de RM). L'activation du cortex moteur et prémoteur serait le reflet de la planification et de l'exécution de la réponse tandis que celle de l'aire motrice supplémentaire correspondrait à la transformation spatiale de l'image mentale.

\subsection{RM de segments corporels et d'outils manipulables}

Suite à la diversité des études sur la relation entre RM de figures abstraites et les fonctions motrices, Parsons (1994) a étudié la RM de segments corporels. Elle est effectuée, soit en tant que telle (morphocinèse), soit pour atteindre un but spatial (téléocinèse), en imaginant que le mouvement du corps se sert d'un objet. Parsons (1994) a demandé aux participants d'identifier si la figure d'une main était celle d'une main droite ou gauche, la présentation se faisant sous des angles variés. La durée nécessaire pour répondre était significativement plus importante lorsque la rotation devait placer la main dans une position difficile à exécuter réellement. Petit et al. (2003) ont comparé la RM d'un membre supérieur à la RM d'un marteau, en intégrant une difficulté supplémentaire, le fait que les images présentées étaient dans des positions possibles ou impossibles. La RM des deux stimuli est directement influencée par l'angle de rotation, confirmant ainsi les résultats de RM d'images abstraites. De même, les temps de réponse pour la RM du segment corporel étaient plus longs que ceux de la RM du marteau. Ce résultat s'explique par le fait que la RM du segment corporel devait nécessairement tenir compte des contraintes anatomiques. Elles sont déterminées par l'architecture 
corporelle et, en conséquence, la vitesse de RM a été réduite. La durée nécessaire pour effectuer la $\mathrm{RM}$ du marteau en condition impossible a augmenté ce qui viendrait du fait que, même si le marteau ne présente pas de contraintes anatomiques et biomécaniques, il reste un outil implicitement lié au mouvement du segment corporel impliqué dans son utilisation (la main en l'occurrence). Aussi, la durée de la RM serait-elle affectée de manière identique à celle du segment corporel. Enfin, la vitesse de RM était plus élevée lorsque le mouvement des mains était possible, ce qui semble logique. Confirmant les hypothèses de Johnson (2000), Petit et al. (2003) ont conclu que la RM de segments corporels suivait les mêmes lois que celle d'objets et que les contraintes biomécaniques influençaient le mouvement des segments impliqués dans leur manipulation.

Plus récemment, Petit et al. (2006) ont étudié l'activité cérébrale lors de la RM d'objets manipulables (marteau) présentés selon des orientations habituelles ou inhabituelles et non fonctionnelles. Dans le premier cas, l'évocation mentale et la RM de la main saisissant l'objet était facile, alors que dans le second, il y avait des problèmes anatomiques et fonctionnels. L'activation cérébrale des systèmes moteurs était plus marquée pour les orientations habituelles. Elle était effective dès la présentation du stimulus, avant même le début de la rotation. Un objet manipulable a donc des caractéristiques motrices intégrées à sa représentation sémantique. Tout objet présenté avec une orientation normale impliquera une activation automatique des programmes moteurs associés à son utilisation, même lorsqu'aucun mouvement n'est exécuté ou planifié. Ce résultat rejoint celui de Decety et al. (1997) qui ont observé une activation des régions motrices de la programmation et l'exécution du mouvement, à partir de la simple observation du mouvement des mains avec l'intention de l'imiter.

Overney et al. (2005) ont étudié l'activité cérébrale lors de la RM de segments corporels dans des positions anatomiquement et biomécaniquement possibles ou impossibles. Le temps de réaction était plus faible pour les positions impossibles. Par ailleurs, la détection d'une position anatomiquement impossible aurait lieu avant le début de la RM et impliquerait l'activation des régions sous-corticales, notamment les amygdales, impliquées dans la réponse émotionnelle douloureuse induite par la vue d'un segment corporel déformé. L'influence de la position du segment que l'on doit mobiliser mentalement a été étudiée par Ionta et al. (2007) pour les mains et les pieds. Les participants devaient indiquer si la figure présentée correspondait à un segment droit ou gauche. Dans une première condition, ils devaient répondre en gardant leurs mains sur leurs genoux alors que dans la seconde, ils devaient les croiser derrière le dos, position inconfortable et inhabituelle. La RM des mains est influencée par les différentes postures, mais pas celle des pieds. Elle était aussi plus rapide lorsque les mains étaient dans une position naturelle, mais cet effet n'a pas été observé pour les pieds. La posture d'un segment corpo- rel donné peut donc influencer sa RM, les informations posturales et de position étant déterminantes dans la construction de leur représentation mentale. Ces résultats confirment ceux de Funk et al. (2005), qui ont également observé un effet de la position des mains, chez des enfants de 5 à 6 ans et chez les adultes. Les informations proprioceptives influencent l'imagerie motrice (Vargas et al., 2004) particulièrement lors de la rotation de segments corporels.

Les travaux mentionnés dans cette partie se sont focalisés sur la RM de segments corporels ou d'outils manipulables. Recourir à de tels stimuli incite fortement les participants à imaginer le mouvement nécessaire pour utiliser ou saisir l'objet, ce qui implique, de fait, des processus moteurs. Comparativement aux RM de structures géométriques informelles et peu familières ( $\mathrm{S}-\mathrm{M}$ cubes), l'imagination des transformations spatiales paraît facilitée. L'expérience d'Amorim et al. (2006) constitue une approche intermédiaire : des caractéristiques humaines ont été données aux formes abstraites originales de Shepard et Metzler. À travers 6 expériences, ils ont montré qu'ajouter « une tête » aux S-M cubes, pour évoquer une posture humaine, facilitait l'incorporation du schéma corporel à la forme abstraite, accélérant ainsi la $\mathrm{RM}$ et rendant sa comparaison au modèle plus précise. La comparaison de la RM d'une posture humaine à celle d'objets (S-M cubes ou lampadaire) ayant une configuration spatiale identique a montré que la transformation spatiale d'un corps humain s'effectuait de façon globale, alors qu'elle était plutôt réalisée par étapes pour les formes abstraites et les objets. Finalement, ils ont observé que lorsqu'une posture ne pouvait pas être simulée par le système sensori-moteur, pour des raisons de contraintes anatomiques et biomécaniques, sa RM ne présentait plus l'avantage d'être intégrée au schéma corporel. Cela explique les durées de traitement prolongées lors des RM de figures impossibles.

\subsection{Stratégie interne vs. Stratégie externe}

Dans quelle situation les participants s'imaginent-ils une RM de l'objet, ou de la force qu'on lui applique (rotation de la main tenant l'objet, par exemple)? Autrement dit, sont-ils acteurs ou observateurs? Kosslyn et al. (1998) ont ainsi comparé la RM de mains à celle de S-M cubes avec comme variable dépendante, les variations hémodynamiques cérébrales (TEP). Ils ont observé une activation des lobules pariétaux supérieurs et inferieurs et du cortex visuel extra-strié lors de la RM des cubes, alors que celle des mains a davantage activé, en plus des structures précédentes, les régions pré-motrices et motrices ainsi que le cortex visuel primaire. Les processus moteurs seraient donc actifs lors de la RM de segments corporels, confirmant ainsi l'existence d'une stratégie consistant à imaginer le mouvement dudit segment corporel, par opposition à la RM de l'objet lui-même. Kosslyn et al. (1998) proposent que la stratégie interne préside à la RM 
des mains, les sujets imaginant mentalement le mouvement de leur propre main pour la comparer au stimulus. Elle n'est pas utilisée uniquement pour la RM de segments corporels, mais aussi durant celle d'objets familiers qu'on peut facilement associer aux mouvements requis pour les manipuler. La stratégie externe fut au contraire privilégiée pour la RM des cubes, cette tâche ne nécessitant pas de manipuler l'objet. Au contraire, les S-M cubes qui sont des figures abstraites difficiles à manipuler mentalement incitent les sujets à utiliser une stratégie externe, indépendante de leurs propres mouvements. Cette hypothèse a été confirmée par Kosslyn et al. (2001). Lorsque les sujets étaient explicitement amenés à utiliser une stratégie interne ou externe pour faire tourner les S-M cubes, le cortex moteur primaire n'était activé que dans le premier cas. Les sujets peuvent donc choisir volontairement une stratégie pour effectuer la RM, qui dépend des consignes ou du type de stimulus.

Vingerhoets et al. (2002) ont étudié l'activité cérébrale durant la RM de figures de mains et celle d'outils manipulables (crayon, taille-crayon, louche, ouvreboîte). Contrairement aux expériences mentionnées précédemment, ils ont observé une activation bilatérale des régions pariétales supérieures, des aires visuelles extra-striées et des régions pré-motrices, indépendamment de la tâche. La stratégie interne peut donc être utilisée pour les deux types de stimuli, surtout pour ceux qui peuvent facilement être intégrés au schéma corporel. La RM de mains et d'outils est toutefois restée plus rapide que celle de formes abstraites, laissant supposer que la stratégie interne donne un avantage par rapport aux stimuli qui impliquent spontanément la stratégie externe.

D'intéressantes données émergent également des études psychophysiques effectuées sur les patients porteurs de lésions unilatérales du cerveau. Tomasino et al. (2003) et Tomasino \& Rumiati (2004) ont notamment comparé les temps de réaction (lors de l'utilisation de la stratégie interne ou externe) de patients ayant une lésion unilatérale gauche à ceux porteurs d'une lésion dans l'hémisphère droit. Ils ont observé que ces derniers avaient davantage de difficultés lors de tâches nécessitant l'utilisation de la stratégie externe, contrairement aux patients atteints d'une lésion dans l'hémisphère gauche, qui présentaient des difficultés pour la stratégie interne. Selon ces auteurs, l'hémisphère gauche interviendrait donc durant la RM de stimuli représentant des mains (stratégie interne).

La stratégie interne est-elle pour autant majoritairement utilisée pour la RM de segments corporels et d'outils, alors que la stratégie externe le serait pour les formes abstraites? Wraga et al. (2003) ont supposé un possible transfert des stratégies motrices activées lors d'une RM de figures de mains à celle de $\mathrm{S}-\mathrm{M}$ cubes. Deux groupes ont été testés, le premier ayant effectué des RM de figures de mains suivies de RM de cubes S-M, alors que le second n'a effectué que des RM de cubes S-M. En comparant l'activité cérébrale des deux groupes durant la RM des cubes, l'activation des régions motrices n'a été observée que dans le premier. Les stratégies motrices utilisées pour la RM de segments corporels pourraient donc être transférées à la RM d'objets abstraits non manipulables. Kawamichi et al. (2007) ont récemment confirmé ces données, tout en ouvrant de nouvelles perspectives sur la différence entre RM d'objets en $2 \mathrm{D}$ et $3 \mathrm{D}$. En effet, ils ont rapporté une activation du lobule pariétal supérieur droit pour les RM en $2 \mathrm{D}$ et des régions prémotrices dorsales de l'hémisphère droit pour les rotations en 3D. L'activation du cortex prémoteur serait donc associée à la visualisation des faces cachées du stimulus visuel, indispensable pour les RM en 3D. Les stratégies visuelle et motrice s'appliqueraient respectivement pour les rotations $2 \mathrm{D}$ et $3 \mathrm{D}$. L'hypothèse d'un tel transfert est séduisante, bien que Bode et al. (2007) aient affirmé que l'activation du cortex moteur primaire est dépendante du type de stratégie employé. Comparant l'activité cérébrale durant la RM de figures abstraites (S-M cubes, formes 2D), d'outils manipulables (calculatrice) et de segments corporels (mains), ils ont observé une activation systématique du cortex moteur primaire. De plus, les participants ont rapporté avoir utilisé la stratégie externe indépendamment du stimulus, afin d'identifier si les deux figures présentées simultanément étaient identiques ou différentes. Ces données confirment partiellement les résultats de Zacks et al. (2002) selon lesquels la stratégie externe était utilisée pour des RM de segments corporels. Selon Bode et al. (2007), la RM en elle-même activerait les régions motrices, celles-ci pouvant diffuser l'activation à des régions corticales adjacentes, comme le cortex prémoteur et pariétal postérieur, puis vers le cortex moteur primaire, ces régions étant interconnectées anatomiquement et fonctionnellement.

Le choix de l'une des deux stratégies peut être précisé grâce aux études comportementales. Zacks et al. (2002) ont démontré que lors d'une RM d'un segment corporel, la stratégie externe pouvait aussi être employée efficacement. Dans un premier temps, les participants devaient reconnaître si deux segments corporels présentés simultanément étaient identiques ou différents. Ensuite, ils devaient indiquer si l'image était un bras droit ou gauche en extension. Dans le premier cas, la durée du traitement était corrélée positivement à l'augmentation de la disparité entre les stimuli, ce qui prouve que les sujets ont effectué une transformation spatiale centrée sur l'objet : ils ont donc eu recours à une stratégie externe pour tourner le stimulus autour de son propre axe de rotation, pour le comparer à la référence. Cela demande plus de temps quand la disparité entre les stimuli est importante. Dans le second cas, en revanche, aucun effet de l'angle de rotation n'a été rapporté. Au cours de la deuxième tâche, les sujets ont choisi la stratégie interne pour reconnaître un bras gauche d'un bras droit. De'Sperati \& Stucchi (2000) affirment qu'on peut avoir recours aux deux stratégies pour un même stimulus. Ils ont d'abord demandé aux participants (tous droitiers) de préciser si un tournevis, présenté avec une flèche indiquant le sens de sa rotation, vissait ou dévissait. Ensuite, ils devaient affirmer si un 
tournevis, placé perpendiculairement au centre du cercle d'une montre et agissant sur ses aiguilles, indiquait un sens de rotation horaire ou antihoraire. Les orientations pour lesquelles la préhension du tournevis est difficile avec la main droite ont nécessité des durées de traitement plus importantes pour la première condition, alors qu'elles n'étaient pas affectées par l'orientation du tournevis pour la seconde. Les sujets ont donc d'abord utilisé une stratégie interne en simulant le mouvement de leur propre main pour manipuler le tournevis, alors que dans la seconde, ils ont privilégié une stratégie externe.

Finalement, la stratégie interne présente-t-elle un avantage par rapport à l'externe? Bien que les résultats soient équivoques, ils semblent à l'avantage de la stratégie interne lorsque le stimulus est un objet manipulable ou un segment corporel. Ceci n'a toutefois pas été retrouvé dans tous les plans de l'espace. Carpenter \& Proffitt (2001) ont comparé les RM nécessitant d'effectuer des transformations spatiales du corps par rapport à un environnement fixe (repérage égocentrique) aux RM requérant de faire tourner mentalement l'environnement par rapport au corps, dans les 3 plans de l'espace. La RM du corps était plus rapide, mais uniquement dans un plan horizontal, comparativement aux plans frontal et sagittal. Le recours à une ou l'autre stratégie dépend alors également du contexte.

\subsection{Interférences entre RM et rotation manuelle simultanée}

Une série d'études vient à l'appui de la neuroimagerie pour préciser l'implication de processus moteurs lors de la RM. Wohlschläger \& Wohlschläger (1998) ont montré qu'une RM de figures abstraites simultanément à une rotation manuelle était plus rapide lorsqu'elles avaient lieu dans la même direction, et inversement. L'interférence entre rotation mentale et manuelle témoigne, indirectement, d'une relation entre l'exécution de la rotation et sa simulation mentale. En conséquence, les activités sportives nécessitant des rotations physiques de la totalité ou d'une partie du corps pourraient bénéficier d'un entraînement intégrant la RM. Wexler et al. (1998) ont confirmé ces données en montrant qu'on était plus rapide tout en commettant moins d'erreurs quand les deux rotations simultanées étaient compatibles. De plus, la durée de la RM était positivement corrélée à la direction et la vitesse de la rotation manuelle. Les auteurs ont conclu que la RM nécessitait une planification et une anticipation motrice et qu'elle était guidée par l'anticipation visuo-motrice. Windischberger et al. (2003) l'ont confirmé en enregistrant une activation bilatérale de l'aire motrice supplémentaire antérieure pendant la RM.

Pour valider cette hypothèse, Wohlschläger (2001) a évalué l'interférence entre la planification d'une rotation manuelle et la RM d'images abstraites. Le protocole consistait à présenter un stimulus indiquant le sens de la $\mathrm{RM}$, tandis que la réponse à ce dernier devait s'effectuer lorsqu'elle se terminait. Ainsi, l'ordre des tâches était : consigne indiquant le sens de la rotation manuelle suivie de la RM d'un stimulus et de la réponse motrice manuelle. La RM était donc effectuée entre la présentation du sens de la rotation manuelle et le début de mouvement de la main, coïncidant avec la planification de la rotation manuelle. Les résultats ont montré que la planification du mouvement de la main interférait avec la RM, seulement dans le cas où les deux axes de rotation étaient parallèles. De plus, la planification de la rotation de la main seule, a été suffisante pour créer l'interférence avec la RM, l'exécution du mouvement n'étant pas indispensable. En d'autres termes, le fait de garder une opération spatiale en mémoire de travail peut interférer avec l'exécution d'une autre opération spatiale comme la RM. Finalement, cette étude a posé l'hypothèse d'une interférence entre le but de l'action réelle (le mouvement de la main) et celui de l'action imaginée, ce qui confirme que la RM implique d'imaginer l'action puis de planifier la rotation.

\section{Effets du sexe et de l'expérience}

La différence entre les capacités individuelles de RM chez les hommes et les femmes a fait l'objet d'un très grand nombre de publications. Les hommes sont majoritairement meilleurs que les femmes (e.g. Linn \& Petersen, 1985 ; Peters et al., 1995; Peters, 2005; Vandenberg \& Kuse, 1978; Voyer et al., 1995; Guillot et al., 2007a). Si ces différences sont multi-causales, deux hypothèses principales ont été défendues l'une biologique et l'autre sociologique. La première s'appuie sur des différences physiologiques et hormonales (Hampson, 1990; Annett, 1992 ; Hooven et al., 2004; Schoning et al., 2007) et des configurations d'activations cérébrales différentes (Jordan et al., 2002). Chez la femme le gyrus intrapariétal, les lobules pariétaux supérieurs et inférieurs, le gyrus temporal inférieur et les régions prémotrices sont activés bilatéralement durant la RM. Chez l'homme les activations s'étendent aux gyrus pariéto-occipital droit, intrapariétal gauche ainsi qu'au lobule supérieur pariétal gauche. En plus des régions prémotrices activées chez les femmes, les hommes ont montré une activation remarquable du cortex moteur gauche. D'après les auteurs, ces différences sont notamment liées à la stratégie, les femmes privilégiant l'identification des différentes parties du stimulus alors que les hommes seraient plutôt sensibles aux caractéristiques de surface. Une autre explication serait que les hommes auraient une stratégie égocentrique, ce qui expliquerait l'activation du cortex moteur primaire. Peters et al. (2007) ont même trouvé une différence de performance liée à l'orientation sexuelle, les hommes hétérosexuels étant plus performants que les homosexuels alors que les femmes homosexuelles étaient plus performantes que les hétérosexuelles. La psychologie sociale attribue davantage cette différence à l'éducation et aux expériences acquises au cours de l'enfance. Les activités pratiquées par les garçons seraient plus favorables au 
développement des capacités spatiales (voitures, robots, lego ${ }^{\circledR} .$. ), que celles des filles (poupée, dinette...), qui laissent moins de place à la manipulation des informations visuospatiales (Baenninger \& Newcombe, 1989; Brosnan, 1998). Alington et al. (1992), Kass et al. (1998) et Roberts \& Bell (2000) ont néanmoins avancé que cette différence pouvait être réduite après un entrainement spécifique des capacités visuo-spatiales et une familiarisation au travail informatique. Récemment, Wiedenbauer \& JansenOsmann (2008) ont montré que l'entraînement des enfants à la rotation manuelle d'un stimulus améliorait leur capacité de RM tout en éliminant les différences liées au genre. De plus, d'autres travaux ont également mis en évidence l'impact des jeux vidéos, privilégiés par les garçons (Griffiths et al., 2004), le simple fait de jouer au Tetris ${ }^{\circledR}$ ou au Blockout ${ }^{\circledR}$ améliore ainsi la performance en RM (De Lisi \& Cammarano, 1996; Okagaki \& Frensch, 1994; Quaiser-Pohl et al., 2006). Finalement cette différence entre hommes et femmes résulte sans doute de l'interaction des deux facteurs.

L'expérience visuo-spatiale serait principalement acquise à travers l'éveil sensori-moteur. Un enfant jouant avec des cubes fait des opérations dans lesquelles il effectue des transformations spatiales des pièces dans les 3 plans de l'espace. Le même type de raisonnement est sollicité durant les jeux du type Tetris ${ }^{\circledR}$, où l'enfant doit anticiper mentalement la position des cubes pour les encastrer les uns dans les autres. Les activités physiques et sportives pratiquées sont également susceptibles d'influencer les capacités visuospatiales : le sportif est souvent amené à anticiper et imaginer l'évolution de la situation sur la base d'informations spatiales, par exemple pour localiser un partenaire ou un adversaire (sport collectifs), la cible à atteindre, se repérer par rapport à un point fixe pour effectuer des rotations (danse, gymnastique...). Le sport a ainsi été considéré comme un facteur favorable d'amélioration des capacités de RM. Naito (1994) et Ozel et al. (2004) ont montré que les sportifs étaient plus performants que les non-sportifs dans les tests de RM, confirmant les résultats de Dror et al. (1993) qui soulignaient la supériorité des pilotes d'avion par rapport aux non-pilotes. Ces résultats en faveur des sportifs sont toutefois équivoques. Plus récemment, Guillot et al. (2007b) n'ont pas trouvé de différence entre sportifs et non-sportifs dans la capacité à faire tourner mentalement des formes géométriques abstraites. Il n'y aurait donc pas de transfert systématique entre la capacité à effectuer des rotations corporelles et la RM.

Une autre forme d'expérience susceptible d'améliorer la RM est liée aux exigences visuo-spatiales de l'activité professionnelle (comme les architectes et les chirurgiens). Dans le domaine médical, la manipulation d'une fibre optique durant une coloscopie ou le placement d'une prothèse de hanche en orthopédie nécessitent une bonne capacité de RM. Plusieurs études ont ainsi souligné l'importance des capacités visuo-spatiales dans l'acquisition des connaissances en anatomie et leur éventuelle application chirurgicale (Rochford, 1985; Garg et al., 2001;
Hegarty et al., 2008; Wanzel et al., 2007; Guillot et al., 2007a; Hoyek et al., 2009). Les chirurgiens obtiennent de très bons scores dans les tests de représentation spatiale et de RM (Risucci, 2002). Wanzel et al. (2002, 2003) et Keehner et al. (2004) ont montré que les étudiants en médecine ayant des capacités visuo-spatiales élevées, apprenaient plus rapidement les techniques chirurgicales complexes que les autres. Mais, en retour, la pratique de la chirurgie les améliorerait d'où l'hypothèse d'un transfert positif entre RM et expérience professionnelle. Peu nombreuses sont les études sur le transfert des capacités visuo-spatiales, suite à un entraînement. Nous avons mis en évidence un transfert positif de la capacité de RM vers l'acquisition de connaissances en anatomie humaine (Hoyek et al., 2009). Après avoir entrainé un groupe d'étudiants STAPS à la RM puis comparé leurs résultats au test VMRT et à l'examen d'anatomie à ceux d'un groupe contrôle, nous avons montré que leur score au VMRT s'était amélioré et qu'il s'était même transféré à l'examen d'anatomie avec un résultat meilleur que celui du groupe contrôle.

Le transfert des capacités de RM vers d'autres domaines d'expérience pourrait se produire si elles sont travaillées et favoriser l'apprentissage de tâches motrices, en particulier dans les activités qui nécessitent une bonne représentation spatiale.

\section{Synthèse et conclusion}

Les relations entre RM et processus moteurs restent controversées, notamment à travers l'implication des réseaux nerveux activés. L'activation des régions motrices est-elle directement liée à des processus moteurs intervenant lors de la résolution des problèmes, ou bien est-elle le reflet de la planification et l'exécution d'un mouvement (action du doigt) lors de la réponse au stimulus? S'il reste probable que l'activation du cortex moteur primaire résulte de la planification motrice, celle du cortex pré-moteur et de l'aire motrice supplémentaire serait due à la RM elle-même. Les contradictions relevées pourraient s'expliquer par le fait que de nombreuses études n'ont pas considéré la dissociation des quatre phases de la RM. L'activation du cortex moteur primaire serait due à l'action du doigt sur le bouton de réponse et ne dépendrait pas directement de la RM au sens strict (Windischberger et al., 2003).

Les expériences impliquant rotations mentales et manuelles n'ont pas pu confirmer si les interférences persistent lorsque la seconde tâche n'implique aucune rotation physique. En d'autres termes, y aurait-il une interférence entre RM et mouvement linéaire de la main ou d'une autre partie du corps? Ou entre RM et une tâche mentale n'exigeant pas de transformation spatiale, comme le calcul mental? Ces questions restent à élucider pour savoir si cette interférence est vraiment due à l'exécution de deux tâches partageant des mécanismes 
de contrôle commun ou bien si c'est le cerveau qui n'arrive pas à analyser et traiter les deux tâches en parallèle?

En revanche, les résultats sont moins équivoques lorsque l'on compare la RM de segments corporels et d'outils familiers et témoignent d'une forte implication des processus moteurs. La capacité de RM d'un segment corporel est étroitement liée au schéma corporel, défini comme la combinaison des sensations, perceptions, mémoires et représentations relatives au corps (Berlucchi \& Aglioti, 1997). Dans ce cas, la stratégie interne (ou egocentrique) présente un avantage par rapport à l'externe, la RM de mains et d'outils étant plus rapide que celle des formes abstraites (Vingerhoets et al., 2002). La question de l'origine de ce choix reste posée. En d'autres termes, quels sont les facteurs induisant une stratégie interne ou externe? Les exigences de la tâche constituent le facteur principal, notamment la nature du stimulus. La RM d'objets s'effectue selon deux cadres de référence, l'un centré sur l'objet et l'autre sur l'environnement, avec les trois plans de l'espace dans lequel l'objet évolue. En effectuant une RM, on établit une relation de couplage entre eux. Si la tâche consiste à comparer deux objets, on procède à un alignement de leurs cadres intrinsèques respectifs et la comparaison se fait dans un environnement commun. Cependant, pour des segments corporels ou des objets manipulables, un nouveau cadre de référence, manuel ou corporel entre en jeu. Quand on bouge un objet avec la main, un couplage de leurs 2 cadres de référence est effectué, qui résulte en leur intégration pour que l'objet et la main qui le tient évoluent dans un environnement commun. Cela suppose une stratégie interne qui active les régions prémotrices. Finalement les caractéristiques individuelles des participants doivent aussi être considérées comme facteur d'influence, les adultes ayant une expérience importante de maniement d'objets, d'où le recours à la simulation de leur propre main en action.

Finalement, si les facteurs déterminant les relations entre RM et processus moteurs, sont difficiles à identifier nous pouvons néanmoins les répartir en six catégories :

(a) le stimulus expérimental, les S-M cubes, lettres, chiffres, segments corporels ou encore les outils étant les plus fréquemment étudiés. Chacun d'eux induirait une activation différenciée des systèmes moteurs et une vitesse de RM différente;

(b) la stratégie adoptée par le sujet, qui peut s'imaginer acteur, c'est-à-dire saisissant et manipulant l'objet ou, au contraire, imaginer l'objet tourner sous l'effet d'une force externe. Dans le premier cas, la RM constitue une forme particulière, d'imagerie motrice. Dans le second, il résulte davantage d'une imagerie visuelle. Comme l'ont récemment montré Guillot et al. (2009), en comparant imagerie visuelle et kinesthésique d'une séquence de mouvements des doigts, les réseaux nerveux impliqués sont partiellement différents, la stratégie interne impliquant davantage les systèmes moteurs; (c) les consignes qui influencent sélectivement la stratégie adoptée et la vivacité des représentations mentales. Elles doivent donc être contrôlées minutieusement à l'aide d'un script d'imagerie ;

(d) les exigences de la RM, car comparer deux stimuli présentés simultanément en faisant tourner l'un d'eux n'implique pas les mêmes opérations qu'une tâche de latéralité (identification de mains gauches ou droites, par exemple) ;

(e) les contraintes géométriques de la RM car une RM en $2 \mathrm{D}$ ou $3 \mathrm{D}$ peut donner des résultats différents, de même en fonction du plan dans lequel on l'effectue;

(f) les différences individuelles et l'expertise : les capacités visuo-spatiales et l'expérience relative à la RM influencent la manière dont le problème est traité. Certaines personnes font tourner mentalement l'objet dans sa globalité, alors que d'autres procèdent plutôt à une RM analytique (segmentaire ou pièce par pièce). La première se caractérise ainsi par un temps de réponse plus faible. De même, l'expérience professionnelle (e.g. chirurgiens, ingénieurs, pilotes) et le niveau de pratique des sportifs influencent aussi la capacité de RM et induisent la stratégie utilisée. La latéralité joue aussi un rôle, un droitier répondant plus rapidement à un stimulus représentant une main droite qu'un gaucher, et inversement.

Pour conclure, les perspectives de recherche en RM et ses relations avec les processus moteurs restent nombreuses et devraient permettre d'élucider à plusieurs questions encore sans réponse. En premier lieu, il faut vérifier s'il existe des interférences entre deux RM simultanées de nature différente, ou encore entre la RM d'un objet et un mouvement corporel n'impliquant aucune rotation. Nous pouvons aussi poser l'hypothèse que les configurations d'activation cérébrale sont différentes chez des personnes ayant une capacité de RM élevée, comparativement à celles ayant des difficultés dans cet exercice. Enfin, même si l'effet de la pratique sportive sur la capacité de RM n'est pas encore définitivement établi, la question de savoir si les sportifs ont de meilleures capacités de RM lorsqu'ils sont spécialistes d'activités nécessitant de maîtriser des rotations corporelles reste posée. Inversement, il faudrait aussi étudier le transfert de compétences en RM (Hoyek et al., 2009), afin de savoir si l'entraînement permet de construire une meilleure représentation mentale du corps en mouvement.

\section{Bibliographie}

Alington, D.E., Leaf, R.C., \& Monaghan, J.R. (1992). Effects of stimulus color, pattern, and practice on sex differences in mental rotations task performance. Journal of Psychology, 126, 539-553.

Alivisatos, B., \& Petrides, M. (1996). Functional activation of the human brain during mental rotation. Neuropsychologia, 35, 111-118. 
Amorim, M.A., Isableu, B., \& Jarraya, M. (2006). Embodied Spatial Transformations: Body Analogy for the Mental Rotation of Objects. Journal of Experimental Psychology, 135, 327-347.

Annett, M. (1992). Spatial ability in subgroups of left- and right-handers. British Journal of Psychology, 83, 493-515.

Baenninger, M., \& Newcombe, N. (1989). The role of experience in spatial test performance: A meta analysis. Sex Roles, 20, 327-345.

Band, G.P.H., \& Kok, A. (2000). Age effects on response monitoring in a mental-rotation task. Biological Psychology, 51, 201-221.

Barnes, J., Howard, R.J., Senior, C., Brammer, M., Bullmore, E.T., Simmons, A., Woodruff, P., \& David, A.S. (2000). Cortical activity during rotational and linear transformations. Neuropsychologia, 38, 1148-1156.

Berlucchi, G., \& Aglioti, S.M. (1997). The body in the brain: neural bases of corporeal awareness. Trends in Neuroscience, 20, 560-564.

Bode, S., Koeneke, S., \& Jäncke, L. (2007). Different strategies do not moderate primary motor cortex involvement in mental rotation: a TMS study. Behavioral and Brain Functions, 3, 38.

Brosnan, M.J. (1998). Spatial ability in children's play with lego blocks. Perceptual and Motor Skills, 87, 19-28.

Carpenter, M., \& Proffitt, D.R. (2001). Comparing viewer and array mental rotations in different planes. Memory and Cognition, 29, 441-448.

Cohen, M.S., Kosslyn, S.M., Breiter, H.C., DiGirolamo, G.J., Thompson, W.L., Anderson, A.K., Bookheimer, S.Y., Rosen, B.R., \& Belliveau, J.W. (1996). Changes in cortical activity during mental rotation. A mapping study using functional MRI. Brain, 119, 89-100.

Cooper, L.A., \& Shepard, R.N. (1973). Chronometric studies of the rotation of mental images. In G. Chase (Ed.), Visual Information Processing (pp. 75-176). New York : Academic Press.

Decety, J., Grezes, J., Perani, D., Jeannerod, M., Procyk, E., Grassi, F., \& Fazio, F. (1997). Brain activity during observation of actions: Influence of action content and subject's strategy. Brain, 120, 1763-1777.

De Lisi, R., \& Cammarano, D.M. (1996). Computer Experience and Gender differences in Undergraduate Mental Rotation Performance. Computers in Human Behavior, 12 (3), 351-361.

De'Sperati, C., \& Stucchi, N. (2000). Motor imagery and visual event recognition. Experimental Brain Research, 133, $273-278$

Dror, I.E., Kosslyn, S.M., \& Waag, W.L. (1993). VisualSpatial Abilities of Pilots. Journal of Applied Psychology, 78, 763-773.

Dror, I.E., \& Kosslyn, S.M. (1994). Mental Imagery and Aging. Psychology and Aging, 9 (1), 90-102.

Funk, M., Brugger, P., \& Wilkening, F. (2005). Motor processes in children's imagery: the case of mental rotation of hands. Developmental Science, 8, 402-408.
Garg, A.X., Norman, G., \& Sperotable, L. (2001). How medical students learn spatial anatomy. Lancet, 35\%, 363-364.

Griffiths, M.D., Davies, M.N., \& Chappell, D. (2004). Demographic factors and playing variables in online computer gaming. Cyberpsychology and Behavior, 4, 479-487.

Guillot, A., Champely, S., Batier, C., Thiriet, P., \& Collet. C. (2007a). Relationship Between Spatial Abilities, Mental Rotation and Functional Anatomy Learning. Advances in Health Sciences Education, 12, 491-507.

Guillot, A., Louis, M., Thiriet, P., \& Collet, C. (2007b). The effects of expertise level and motor skill characteristics on mental rotation, In L.S. Boyar (Eds.) New psychological tests and testing research (pp. 231-241).

Guillot, A., Collet, C., Nguyen, V.A., Malouin, F., Richards, C., \& Doyon, J. (2009). Brain activity during visual versus kinesthetic imagery: An fMRI study. Human Brain Mapping, 30, 2157-2172.

Hampson, E. (1990). Variations in sex-related cognitive abilities across the menstrual cycle. Brain and Cognition, 14, 26-43.

Harris, I.M., Egan, G.F., Sonkkila, C., Tochon-Danguy, H.J., Paxinos, G., \& Watson, J.D.G. (2000). Selective right parietal lobe activation during mental rotation. A parametric PET study. Brain, 123, 65-73.

Hegarty, M., Keehner, M., \& Cohen, C. (2008). The Role of Spatial Cognition in Medicine: Applications for Selecting and Training Professionals, In G. Allen (Ed.), Applied Spatial Cognition. Mahwah, NJ : Erlbaum, pp. 285-317.

Heil, M., \& Jansen-Osmann, P. (2007). Children's left parietal brain activation during mental rotation is reliable as well as specific. Cognitive Development, 22, 280-288.

Hertzog, C., Vernon, M.C., \& Rypma, B. (1993). Age Differences in Mental Rotation Task Performance: The influence of Speed/Accuracy Tradeoffs. Journal of Gerontology, 48 (3), 150-156.

Hoyek, N., Collet, C., Rastello, O., Fargier, P., Thiriet, P., \& Guillot, A. (2009). Enhancement of Mental Rotation Abilities and its Effect on Anatomy Learning. Teaching and Learning in Medicine, 21, 201-206.

Hooven, C.K., Chabris, C.F., Ellison, P.T., \& Kosslyn, S.M. (2004). The relationship of male testosterone to components of mental rotation. Neuropsychologia, 42, 782-790.

Ionta, S., Fourkas, A.D., Fiorio, M., \& Aglioti, S.M. (2007). The influence of hands posture on mental rotation of hands and feet. Experimental Brain Research, 183, 1-7.

Iwaki, S., Ueno, S., Imada, T., \& Tonoike, M. (1999). Dynamic cortical activation in mental image processing revealed by biomagnetic measurement. NeuroReport, 10, 1793-1797.

Jansen-Osmann, P., \& Heil, M. (2007). Developmental aspects of parietal hemispheric asymmetry during mental rotation. Neuroreport, 18, 175-178.

Johnson, S.H. (2000). Thinking ahead: the case for motor imagery in prospective judgements of prehension. Cognition, $74,33-70$. 
Jordan, K., Heinze, H.J., Lutz, K., Kanowski, M., \& Jancke, L. (2001). Cortical activations during the mental rotation of different visual objects. NeuroImage, 131, 143-152.

Jordan, K., Wüstenberg, T., Heinze, H.J., Peters, M., \& Jancke, L. (2002). Women and men exhibit different cortical activation patterns during mental rotation tasks. Neuropsychologia, 4013, 2397-2408.

Kass, S.J., Ahlers, R.H., \& Dugger, M. (1998). Eliminating Gender Differences Through Practice in an Applied Visual Spatial Task. Human Performance, 11, 337-349.

Kawamichi, H., Kikuchi, Y., Noriuchi, M., Senoo, A., \& Ueno, S. (2007). Distinct neural correlates underlying two- and three-dimensional mental rotations using three dimensional objects. Brain Research, 1144, 117-126.

Keehner, M., Tendick, F., Meng, M.W., Anwar, H.P., Hegarty, M., Stoller, M.M., \& Duh, Q.Y. (2004). Spatial ability, experience, and skill in laparoscopic surgery. American Journal of Surgery, 188, 71-75.

Kosslyn, S.M., Digirolamo, G.J., Thompson, W.L., \& Alpert, N.M. (1998). Mental rotation of objects versus hands: Neural mechanisms revealed by positron emission tomography. Psychophysiology, 35, 151-161.

Kosslyn, S.M., Thompson, W.L., Wraga, M., \& Alpert, N.A. (2001). Imagining rotation by endogenous versus exogenous forces: Distinct neural mechanisms. NeuroReport, 12, 2519-2525.

Kucian, K., von Aster, M., Loenneker, T., Dietrich, T., Mast, F.W., \& Martin, E. (2007). Brain activation during mental rotation in school children and adults. Journal of Neural Transmission, 114, 675-686.

Lamm, C., Windischberger, C., Moser, E., \& Bauer, H. (2007). The functional role of dorso-lateral premotor cortex during mental rotation. An event-related fMRI study separating cognitive processing steps using a novel task paradigm. NeuroImage, 36, 1374-1386.

Linn, M., \& Petersen, A.C. (1985). Emergence and characterization of sex differences in spatial ability: A meta-analysis. Child Development, 56, 1479-1498.

Marmor, G.S. (1975). Development of kinetic images: When does the child first represent movement in mental images? Cognitive Psychology, 7, 548-559.

Marmor, G.S. (1977). Mental rotation and number conservation: are they related? Developmental Psychology, 13, $320-325$.

Metzler, J., \& Shepard, R.N. (1974). Transformational studies of the internal representation of three-dimensional objects, In R. Solso (Ed.), Theories in cognitive psychology. The Loyola Symposium (pp. 147-201). Potomac, MD : Erlbaum.

Naito, E. (1994). Controllability of motor imagery and transformation of visual imagery. Perception and Motor Skills, 78, 479-487.

Okagaki, L., \& Frensch, P.A. (1994). Effects of video game playing on measures of spatial performance: Gender effects in late adolescence. Journal of Applied developmental Psychology, 15, 33-58.
Overney, L.S., Michel, C.M., Harris, I.M., \& Pegna, A.J. (2005). Cerebral processes in mental transformations of body parts: Recognition prior to rotation. Cognitive Brain Research, 25, 722-734.

Ozel, S., Larue, J., \& Molinaro, C. (2004). Relation between sport and spatial imagery: comparison of three groups of participants. Journal of Psychology, 138, 49-63.

Parsons, L.M. (1994). Temporal and kinematic properties of motor behavior reflected in mentally simulated action. Journal of Experimental Psychology, 20, 709-730.

Peters, M. (2005). Sex differences and the factor of time in solving Vandenberg and Kuse mental rotation problems. Brain and Cognition, 5\%, 176-184.

Peters, M., Laeng, B., Latham, K., Jackson, M., Zaiyouna, R., \& Richardson, C. (1995). A redrawn Vandenberg and Kuse mental rotations test: Different versions and factors that affect performance. Brain and Cognition, 28, 39-58.

Peters, M., Manning, J.T., \& Reimers, S. (2007). The Effects of Sex, Sexual Orientation, and Digit Ratio (2D:4D) on Mental Rotation Performance. Archives of Sexual Behavior, 36, 251-260.

Petit, L.S., Pegna, A.J., Mayer, E., \& Hauert, C.A. (2003). Representation of anatomical constraints in motor imagery: mental rotation of a body segment. Brain and Cognition, 51, 95-101.

Petit, L.S., Pegna, A.J., Harris, I.M., \& Michel, C.M. (2006). Automatic motor cortex activation for natural as compared to awkward grips of a manipulable object. Experimental Brain Research, 168, 120-130.

Quaiser-Pohl, C., Geiser, C., \& Lehmann, W. (2006). The relationship between computer-game preference, gender, and mental-rotation ability. Personality and Individual differences, 40, 609-619.

Richter, W., Ugurbil, K., Georgopoulos, A., \& Kim, S.G. (1997). Time-resolved fMRI of mental rotation. NeuroReport, 8, 3697-3702.

Richter, W., Somorjai, R., Summers, R., \& Jarmasz, M. (2000). Motor area activity during mental rotation studied by time-resolved single-trial fMRI. Journal of Cognitive Neuroscience, 12, 310-320.

Risucci, D.A. (2002). Visual spatial perception and surgical competence. The American Journal of Surgery, 184, 291295.

Roberts, J.E., \& Bell, M.A. (2000). Sex Differences on a computerized mental rotation task disappear with computer familiarization. Perceptual and Motor Skills, 91, 10271034.

Rochford, K. (1985). Spatial learning disabilities and underachievement among university anatomy students. Medical Education, 1, 13-26.

Schöning, S., Engelien, A., Kugel, H., Schäfer, S., Schiffbauer, H., Zwitserlood, S., Pletziger, E., Beizai, P., Ohrmann, P., Greb, R.R., Lehman, W., Heindel, W., Arolt, V., \& Conrad, C. (2007). Functional anatomy of visuo-spatial working memory during mental rotation is influenced 
by sex, menstrual cycle, and sex steroid hormones. Neuropsychologia, 45, 3203-3214.

Shepard, R.N., \& Metzler, J. (1971). Mental rotation of three dimensional objects. Science, 171, 701-703.

Tomasino, B., \& Rumiati, R.I. (2004). Effects of Strategies on Mental Rotation and Hemispheric Lateralization: Neuropsychological Evidence. Journal of Cognitive Neuroscience, 16 (5), 878-888.

Tomasino, B., Toraldo, A., \& Rumiati, R.I. (2003). Dissociation between the mental rotation of visual images and motor images in unilateral brain-damaged patients. Brain and Cognition, 51, 368-371.

Vandenberg, S., \& Kuse, A. (1978). Mental rotation, a group test of 3-D spatial visualization. Perceptual and Motor Skills, 47, 599-604.

Vargas, C.D., Olivier, E., Craighero, L., Fadiga, L., Duhamel, J.R., \& Sirigu, A. (2004). The Influence of Hand Posture on Corticospinal Excitability during Motor Imagery: A Transcranial Magnetic Stimulation Study. Cerebral Cortex, 14, 1200-1206.

Vingerhoets, G., Santens, P., Van Laere, K., Lahorte, P., Dierckx, R.A., \& De Reuck, J. (2001). Regional Brain Activity during Different Paradigms of Mental Rotation in Healthy Volunteers: A Positron Emission Tomography Study. NeuroImage, 13, 381-391.

Vingerhoets, G., de Lange, F.P., Vandemaele, P., Deblaere, K., \& Achten, E. (2002). Motor imagery in mental rotation: An fMRI study. NeuroImage, 17, 1623-1633.

Voyer, D., Voyer, S., \& Bryden, M.P. (1995). Magnitude of sex differences in spatial abilities: a meta-analysis and consideration of critical variables. Psychological Bulletin, 117, 250-270.

Wanzel, K.R., Hamstra, S.J., Anastakis, D.J., Matsumoto, E.D., \& Cusimano, M.D. (2002). Effect of visual-spatial ability on learning of spatially-complex surgical skills. Lancet, 359, 230-231.

Wanzel, K.R., Hamstra, S.J., Caminiti, M.F., Anastakis, D.J., Grober, E.D., \& Reznick, R.K. (2003). Visual-spatial ability correlates with efficiency of hand motion and successful surgical performance. Surgery, 134, 750-757.

Wanzel, K.R., Anastakis, D.J., McAndrews, M.P., Grober, E.D., Sidhu, R.S., Taylor, K., et al. (2007). Visual-spatial ability and fMRI cortical activation in surgery residents. The American Journal of Surgery, 193, 507-510.

Wexler, M., Kosslyn, S.M., \& Berthoz, A. (1998). Motor processes in mental rotation. Cognition, 68, 77-94.

Wiedenbauer, G., \& Jansen-Osmann, P. (2008). Manual training of mental rotation in children. Learning and instruction, 18, 30-41.

Windischberger, C., Lamm, C., Bauer, H., \& Moser, E. (2003). Human motor cortex activity during mental rotation. NeuroImage, 20, 225-232.

Wraga, M., Thompson, W.L., Alpert, N.M., \& Kosslyn, S.M. (2003). Implicit transfer of motor strategies in mental rotation. Brain and Cognition, 52, 135-143.

Wohlschläger, A. (2001). Mental object rotation and the planning of hand movements. Perception \& Psychophysics, 63, 709-718.

Wohlschläger, A., \& Wohlschläger, A. (1998). Mental and manual rotation. Journal of Experimental Psychology: Human Perception 8 Performance, 24, 397-412.

Zacks, J.M. (2008). Neuroimaging Studies of Mental Rotation: A Meta-analysis and Review. Journal of Cognitive Neuroscience, 20, 1-19.

Zacks, J.M., Mires, J., Tversky, B., \& Hazeltine, E. (2002). Mental spatial transformations of objects and perspective. Spatial Cognition 85 Computation, 2, 315-322. 